\section{Cahiers de littérature orale}

$71 \mid 2012$

Interlocutions périlleuses

\title{
Pipi, caca : comment en parle-t-on chez les Gbaya?
}

\section{Paulette Roulon-Doko}

\section{OpenEdition}

Journals

Édition électronique

URL : https://journals.openedition.org/clo/1464

DOI : $10.4000 /$ clo. 1464

ISSN : 2266-1816

Éditeur

INALCO

\section{Édition imprimée}

Date de publication : 1 janvier 2012

Pagination : 53-78

ISBN : 978-2-85831-206-1

ISSN : 0396-891X

\section{Référence électronique}

Paulette Roulon-Doko, «Pipi, caca : comment en parle-t-on chez les Gbaya ? », Cahiers de littérature orale [En ligne], 71 | 2012, mis en ligne le 17 avril 2014, consulté le 30 juin 2021. URL : http:// journals.openedition.org/clo/1464; DOI : https://doi.org/10.4000/clo.1464

Ce document a été généré automatiquement le 30 juin 2021

\section{(c) (†) 8)}

Cahiers de littérature orale est mis à disposition selon les termes de la Licence Creative Commons Attribution - Pas d'Utilisation Commerciale 4.0 International. 


\title{
Pipi, caca : comment en parle-t-on chez les Gbaya?
}

\author{
Paulette Roulon-Doko
}

Il n'y avait pas pour eux [les Anciens] de choses que
l'on ne puisse dire. Dans Aristophane, on chie sur la
scène. (Flaubert, 1853, 81)

1 En français contemporain la coexistence d'une langue écrite et d'une langue parlée est un terrain propice à la manifestation de différents niveaux de langue. Il n'y a pas en particulier de vocabulaire neutre pour parler de ce qui relève du scatologique, terme formé sur deux termes grecs skôr "excrément ${ }^{1}$ et logos "parole ", signifiant littéralement un 'discours sur les excréments'. Tous les parents parlent du pipi et du caca de leurs enfants dans la vie quotidienne puisque c'est un apprentissage essentiel et, dans la rue en ville, on fait la chasse aux crottes de chien et on se plaint de l'odeur de la pisse qu'ils déposent un peu partout. Les médecins, eux, parlent des selles et de l'urine lorsqu'ils interrogent un malade. Le terme 'merde' est généralement perçu comme vulgaire ou grossier et ceux d'excrément et de fèces plutôt comme des termes scientifiques. Dans une culture de tradition orale où l'opposition entre oral et écrit n'est pas encore intégrée dans la vie quotidienne, comment en parle-t-on?

2 Mon étude présentera le cas d'une société de chasseurs-cueilleurs-cultivateurs, les Gbaya $^{2}$ qui vivent en Afrique Centrale. Dans cette culture, le savoir est commun à tous et chacun peut y avoir également accès. C'est une société égalitaire, sans hiérarchie sociale où la parole circule librement, sans différences marquées en fonction des conditions d'usage possible. Parole quotidienne, récits variés, contes, jugements utilisent un vocabulaire commun à tous y compris pour parler de ce 'domaine' pour lequel il n'y a pas de tabou et dont on parle librement. C'est pourquoi j'ai choisi « pipi » et « caca » comme traduction de base (mot à mot) et dans le titre de cet article, parce que cette façon de dire reste la plus employée dans le domaine familier et de ce fait, hors contexte, est la moins marquée, ni scientifique ni vulgaire et pas nécessairement enfantine. 
3 Je présenterai tout d'abord les termes qui sont à la disposition des locuteurs et leurs emplois courants dans la vie quotidienne et j'étudierai ensuite la conception gbaya de ce domaine par l'étude des représentations qu'ils en ont, recourant pour cela à l'examen des devinettes, des proverbes puis des contes, dont la traduction en français pose de façon récurrente le problème de savoir comment ne pas tomber dans le vulgaire ou le scientifique, ni l'infantilisation.

\section{Le vocabulaire scatologique}

4 D'après le Dictionnaire historique Robert (Rey, 1994), le terme excrément, relevé en 1534 chez Rabelais par emprunt au latin impérial excrementum « déchet » et « excrétion, déjection » de excretus (p.p. de excernere " passer au tamis » et dans la langue médicale " évacuer ») a tout d'abord désigné l'ensemble des matières évacuées du corps (matières fécales, urine, mucus nasal, sueur) avant que son emploi ne soit restreint au sens de «matières fécales ». Dans la conception physiologique des Gbaya, le corps rejette un certain nombre de produits : excréments, urine, larmes, sueur et lymphe, tous issus de l'absorption de nourriture, les distinguant des produits préexistants dans le corps lui-même - lait, sperme et sang - qui ne sont, eux, jamais mis directement en relation avec la nourriture. Ces derniers sont regroupés sous l'appellation de " semences du corps » kpáré béi alors qu'aucune appellation générique ne regroupe les matières évacuées du corps.

5 En gbaya, la catégorie du nom représente plus de la moitié des termes du lexique, tandis que celle du verbe atteint seulement $10 \%$. Cependant, tandis que les verbes peuvent produire des noms, il n'y a aucune attestation d'une production d'un verbe à partir d'un nom, contrairement au français où on peut presque toujours former un verbe à partir d'un nom. Cette situation est bien illustrée par le vocabulaire étudié ici.

\section{Les noms employés}

6 Le nom đ’̀r qui signifie " déchet $^{3}$ » permet de désigner (i) la " merde » (déchet du corps humain ou animal) et aussi (ii) un « résidu», comme la « cire» issue du rayon de miel ou la «gangue» qui entoure le fer au moment de la fonte. Le nom Píni signifie seulement «urine». Aucun de ces deux noms n'est explicable: ce sont des termes simples originaux.

7 Dans le dictionnaire des synonymes (Bertaud du Chazaud, 1971) les entrées 'caca' et 'merde' renvoient à 'excrément' qui est ainsi renseigné :

Excrément: I. De l'homme 1) Méd. ou neutre : crotte, déchet, déjection, excrétion, fèces, flux alvin ${ }^{4}$, garde-robe (vx.), matières, matières alvines/ fécales, méconium ${ }^{5}$ (nouveau-né), selles. 2) Familier ou enfantin : caca, gros, grosse commission, pot. 3) Vulg. ou arg. : bran, brenne, bronze, chiasse, colombin, confiture, étron, foire, marchandise, merde, mouscaille, paquet, pêche, sentinelle. II. Des animaux : bouse, chiure, colombine, crotte, crottin, fient, fiente, fumées (vén.), purin. III. Par ext. : boue, gadoue, immondice, ordure, poudrette, rebut, résidu. (p. 192)

Par contre on y trouve trois entrées 'pipi' 'urine' et 'pisse' :

Pipi : urine, pisse. (p. 326)

Urine : pipi (enf.), pissat, pisse. (p. 456)

Pisse : eau, urine, pipi, pissat. (p. 327) 
Il est frappant que le caca a développé en français un vocabulaire beaucoup plus important que le pipi. L'excrément humain manifeste trois niveaux de langue (i) médical dit neutre, (ii) familier ou enfantin, (iii) vulgaire ou argotique tandis que le pipi a uniquement une mention 'enfantin' lorsqu'il est sous l'entrée 'urine' et rien d'autre. Le Trésor de la Langue Française (TLF) qualifie d'une façon comparable 'pipi' de familier mais ajoute le qualificatif de vulgaire pour 'pisse' et réserve 'pissat' à l'urine des animaux. Sous l'entrée 'caca' il ne donne comme synonymes que : crotte, merde (trivial), déjection, fèces, selles (langage médical).

10 En gbaya, il n'y a rien de comparable. Le terme Ṕinì «pipi » ne réfère qu'à l'urine humaine ou animale. Quant au terme đ’̀̀r " déchet d’une activité », il ne désigne pas seulement, comme cela vient d'être présenté, l'excrément humain ou animal contrairement au terme nàáḟ̌̃ « caca, merde » d'un emploi nettement moins fréquent et presque toujours employé au sein de la collocation «odeur de merde » p̌̌r nááḟ̃ ou $p \check{r}$ đòr. Cette odeur de merde est d'ailleurs lexicalisée par un adjectif-adverbe spécifique hàlò-hàlò. C'est ainsi parce qu'elle « sent la merde " nún hàlò-hàlò (sentir/ ) qu'une apocynacée de forêt est appelée "arbre-merde » tè-d’̀̀r. Enfin le côté sale et dégoûtant du caca est bien manifesté par l'insulte ${ }^{\prime}$ ว̌r tèmé (caca. $\mathrm{D}^{6} /$ corps.D.2s) qu'on pourrait traduire par « merdeux ».

\section{Les verbes employés}

11 Pour désigner l'action de déféquer ou de pisser, c'est en gbaya le même verbe s? qui va être utilisé. Ce verbe exprime la présence d'un frottement produit ou spontané et signifie, construit transitivement (i) « frotter, enduire », (ii) « railler, tourmenter » et (iii) « excréter » avec les noms « caca » et «pipi » comme complément d'objet direct. Construit intransitivement, il signifie "produire une substance (déjection, sécrétion) » et s'applique à la déjection qu'est la diarrhée, à la sécrétion des végétaux et à la formation de monticule de terre par les termites. Il produit deux déverbatifs. L'un formé régulièrement sò̀ ¿ « diarrhée " spécifie une excrétion particulière et l'autre, irrégulier du fait de son schème tonal haut só হ́ « "résine, déjection de terre " a une valeur plus générique et peut être spécifié: sỡ Dó bírí "résine de Canarium schweinfurthii ", sỡ

12 En français, dans le dictionnaire des synonymes déjà cité, on ne trouve aucune entrée pour 'déféquer' et deux entrées pour 'pisser' :

Pisser : I. V.intr 1) Au pr: faire/tomber de l'eau (pop.), faire pipi, pissoter, uriner.

2) Par ext : couler, fuir, suinter. II. V.tr1) Au pr: évacuer, faire, perdre. 2) Fig. compiler, produire, rédiger (p. 327).

Uriner : I. faire pipi, lâcher, tomber de l'eau, pisser, pissoter, se mouiller (p. 456).

Le TLF mentionne 'uriner' et qualifie 'pisser' de vulgaire et 'faire pipi' de familier. Quant à 'déféquer', le TLF en fait une entrée où le sens 'expulser les matières fécales' est donné pour l'emploi intransitif sans mention d'aucun synonyme et fait également une entrée 'chier' caractérisé de trivial et suivi des synonymes 'aller à la selle' et 'faire caca' (familier). Ce n'est que sur Wikipedia qu'on trouve à l'entrée 'aller à la selle', une liste de neuf synonymes : caguer (régionalisme), couler un bronze (familier), chier (vulgaire), déféquer (soutenu), faire caca et faire popo (langage enfantin), (dé)mouler un cake et (dé)poser une pêche (familier) et tartir (argot). 
14 En gbaya, le recours à un seul verbe non spécifique de ces excrétions so en souligne la valeur neutre peu propice à un traitement distinctif de ces deux activités : il n'y a qu'une seule façon d'en parler. Alors qu'en français on doit choisir entre 'uriner et déféquer', 'pisser et chier' ou 'faire pipi et faire caca' et donc adopter un niveau de langue, en l'occurrence soutenu, vulgaire, familier ou enfantin, car il n'y a pas de façon neutre d'en parler.

Parlant des oiseaux, les Gbaya connaissent le rôle joué par leurs fientes dans le processus de dissémination de certaines graines et l'expriment en ces termes :

\begin{tabular}{|l|l|l|l|l|}
\hline 1. & Pó & nóć & nón & wáyáà, \\
& PL & oiseau & INAC.manger & fruit.DEF \\
& Pèé & wà & só & cóà \\
caca.DEF & \\
& puis.D & IP & INAC.excréter & \\
& ká & hòá & & \\
& alors & Acc.sortir & & \\
\hline & Les oiseaux mangent les fruits puis ils en expulsent le caca d'où cela \\
& germera.
\end{tabular}

Pour exprimer la même chose le français écrit en termes savants: “La graine, indigestible, est libérée dans les matières fécales " et dispose du terme d'endozoochorie qui est loin d'être connu de tous.

\section{Les adjectifs employés}

17 Pour exprimer la qualification le gbaya dispose de nombreux adjectifs dont une souscatégorie d'adjectifs-adverbes (AA) qui comprend plus de deux mille termes. De l'urine on ne caractérise que la couleur en recourant au terme dééré « couleur thé doré » qui peut par ailleurs s'appliquer à tout liquide. Par contre, dix-neuf adjectifs-adverbes permettent de caractériser un excrément. Un seul déjà mentionné ci-dessus « odeur de merde " hàlò-hàlò est spécifique. Tous les autres ont un sens qui n'est pas une valeur dédiée, ce qui, de fait, ne les classe pas à l'intérieur d'un registre spécifique comme le fait nécessairement la traduction en français où il faut choisir entre médical ou soutenu pour 'excrément', 'selle', 'fèces', vulgaire ou argotique pour 'crotte ${ }^{8 \text {, , 'étron }}$ ', 'merde'.

\begin{tabular}{|c|c|}
\hline 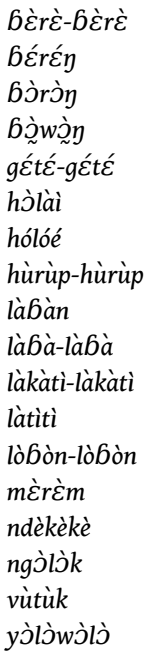 & $\begin{array}{l}\text { en billes } \\
\text { de forme allongée } \\
\text { allongé et pointu } \\
\text { oblong } \\
\text { en une grosse masse molle } \\
\text { mou (impression tactile) } \\
\text { puant (pourriture/excrément) } \\
\text { énorme } \\
\text { qui fait un gros tas mou } \\
\text { qui fait plusieurs tas mous } \\
\text { boueux } \\
\text { qui coule difficilement } \\
\text { chute d'une masse molle } \\
\text { qui glisse } \\
\text { qui fait un monticule } \\
\text { minuscule } \\
\text { qui fait un empilement } \\
\text { trop long }\end{array}$ \\
\hline
\end{tabular}



d'arbres, (ii) du miel, (iii) une nourriture dans la gorge ou (iv) une selle en cas de constipation. Dans tous ces cas est soulignée la difficulté du produit considéré à suivre le chemin qu'il emprunte.

Pour l'urine, la façon dont elle est projetée est exprimée par plusieurs adjectifsadverbes qui, contrairement aux précédents, lui sont majoritairement spécifiques.

\begin{tabular}{|l|l|}
\hline sàa & jet incontrôlé (urine ou vomi) \\
sìrì-sìrìp & par saccades \\
$w \grave{\varepsilon} \grave{c}$ & de façon irrépressible (urine) \\
\hline
\end{tabular}

Le gbaya dispose donc d'un vocabulaire riche permettant d'exprimer toutes sortes de nuances sans, dans la majorité des cas, qu'il s'agisse d'un vocabulaire spécifique. On ne peut donc en gbaya identifier un vocabulaire strictement scatologique.

\section{Que nous apprennent les devinettes?}

21 Le terme "devinette " sì dérive du verbe siy "laisser tomber », la spécifiant comme une 'parole qu'on laisse tomber'. Les devinettes constituent chez les Gbaya un genre littéraire dont le répertoire, plus ou moins figé, est actualisé au cours de chaque séance (Roulon-Doko, 2004). Procédant par la formulation d'images qui manifestent les choix des Gbaya pour exprimer certains domaines conceptuels, elles entraînent la mémoire de chacun qui, au besoin, peut réactualiser certains éléments. Elles sont donc formulées comme une définition dont il convient de trouver le référent le plus vite possible, en tous cas avant les autres. Tandis que pour tous les participants c'est avant tout une distraction collective qui les replonge dans les spécificités de la culture qu'ils partagent, pour l'observateur extérieur c'est un moyen privilégié d'accès à la culture qu'elles manifestent.

Plus de la moitié des devinettes réfèrent au monde humain, ciblant (i) diverses parties du corps et majoritairement le sexe, mais aussi (ii) des productions du corps telles le pipi et le caca qui y occupent une place importante.

Trois devinettes associent «caca et pipi » Còr Pín Pínì, comme un automatisme soulignant leur concomitance fréquente au moment de leur excrétion (verbe so " excréter ») qui est aussi couramment exprimée par l'expression « aller aux toilettes »

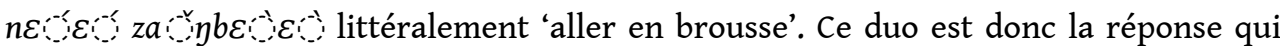
convient aux trois devinettes suivantes :

\begin{tabular}{|l|l|l|l|}
\hline 2. & dònò & zú & nù \\
& marteau_de_forge & INAC.s'enfoncer & terre \\
& rì̀, & ỳ̀ & Acc.faire.D \\
& en_enfonçant_d'un_coup & eau & \\
& bò̀ & & \\
& en_trombe_d'eau & & \\
\hline & Le marteau de forge s'enfonce d'un coup dans la terre, l'eau coule en trombe. \\
\hline
\end{tabular}




\begin{tabular}{|l|l|l|l|}
\hline 3. & wèsé & gòm & gbàdàwà, \\
& jour & à.frapper & \\
& kórò & tèá & \\
& pluie & Acc.venir & \\
\hline & Le jour où on découpe un babouin, il pleut. \\
\hline
\end{tabular}

\begin{tabular}{|l|l|l|l|}
\hline 4. & dònò & yék & nù \\
& marteau_de_forge & INAc.tomber & Gùtù \\
ngb̀̀á & Acc.venir \\
& à_la_renverse & pluie_sp. & \\
\hline & Le marteau de forge est tombé à la renverse, la pluie du matin \\
& tombe.
\end{tabular}

Les éléments de ce duo sont cependant de nature bien différente. Tandis que le caca est défini par son aspect massif que manifestent les images du "marteau de forge » dònò (très lourd bloc de métal), du «babouin " gbàdàwà littéralement 'grand singe' ou de l'« éléphant » fòrò dont l'emploi adjectival signifie 'énorme', la nature fluide du pipi est illustrée par un écoulement spécifié « comme une trombe d'eau » bò̀̀ ou rapportée soit génériquement à la "pluie » kórò soit plus spécifiquement à la "pluie fine » bùtù qui tombe le matin en juin.

Deux devinettes ont pour réponse le caca, l'une reprend l'image de l'éléphant pour rappeler qu'en dépit de sa masse, on l'abandonne sur place :

\begin{tabular}{|c|c|c|c|}
\hline 5. & $\begin{array}{l}\text { mé } \\
2 S \\
\text { pí } \\
\text { INF.ACC.jeter } \\
\text { Pé } \\
\text { INAC.poser } \\
\text { téà, } \\
\text { corps.3S } \\
\text { wár, } \\
\text { chemin }\end{array}$ & 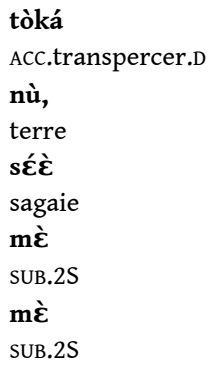 & $\begin{array}{l}\text { fòrò } \\
\text { éléphant } \\
\text { mè } \\
\text { sub.2S } \\
\text { bón } \\
\text { INF.Acc.rester } \\
\text { bá } \\
\text { INAC.prendre } \\
\text { péí } \\
\text { INAC.revenir }\end{array}$ \\
\hline & \multicolumn{3}{|c|}{$\begin{array}{l}\text { Tu as percé un éléphant et tu laisses la sagaie dans son corps puis tu reprends la } \\
\text { route et reviens. }\end{array}$} \\
\hline
\end{tabular}

L'autre associe le caca à la mouche. Cette association est exprimée en termes de relation d'alliance, qui souligne la forte proximité entre le caca et la mouche, ce qu'on retrouve en français commun lorsqu'on parle d'une 'mouche à merde'. La même devinette peut aussi avoir comme réponse le «bousier » náá-kìn-đ’̀r (celui/à rouler/ excrément) un coléoptère qu'on voit souvent roulant une boulette de bouse :

\begin{tabular}{|l|l|l|l|}
\hline 6. & fòr & zí & né \\
& $\begin{array}{l}\text { beau-parent } \\
\text { Pò }\end{array}$ & $\begin{array}{l}\text { mouche } \\
\text { ndé } \\
\text { est-ce-que }\end{array}$ \\
& qui & \\
\hline & Qui est le beau-parent ${ }^{10}$ de la mouche? \\
\hline
\end{tabular}

27 Les deux devinettes suivantes dont la réponse est le pipi mettent en scène comme dans l'exemple 5, une activité de chasse mais ici le gibier n'est pas identifié (ex. 7) ou bien 
c'est un petit rongeur très commun l'« aulacode » bíá (ex. 8). Dans les deux cas la nature du gibier n'importe pas, l'attention est portée sur le déplacement qu'on lui prête en le faisant, dans les deux cas, revenir à son point de départ. C'est de fait la trajectoire suivie par l'urine ${ }^{11}$ qui est ainsi caractérisée puisque les dernières gouttes tombent toujours au plus près de leur point de départ. Ce phénomène valable pour les deux sexes, est encore plus marqué pour les hommes, ce qu'exprime la devinette 8 qui fait parcourir à l'aulacode un vaste territoire entre deux rivières 'Boyanga et Dota et dont la réponse est plus spécifiquement «le pipi des hommes » :

\begin{tabular}{|c|c|c|c|}
\hline 7. & $\begin{array}{l}\text { mà } \\
\text { un_certain } \\
\text { mé } \\
2 S \\
\text { má } \\
\text { INAC.détaler } \\
\text { ká } \\
\text { alors } \\
\text { ká } \\
\text { alors } \\
\text { mé } \\
2 S \\
\text { nèmé } \\
\text { REL.2S. }\end{array}$ & $\begin{array}{l}\text { sàdì } \\
\text { animal } \\
\text { tòká } \\
\text { Acc.piquer } \\
\text { yù } \\
\text { course } \\
\text { kór-zúà } \\
\text { nuque.3S } \\
\text { pèyá } \\
\text { ACc.revenir.D } \\
\text { gbèćà } \\
\text { Acc.tuer.D.3S } \\
\text { tòká } \\
\text { Acc.transpercer }\end{array}$ & $\begin{array}{l}\text { sènẽi, } \\
\text { dedans.ANAPH } \\
\text { ká } \\
\text { alors } \\
\text { síné, } \\
\text { en_allant } \\
\text { néí, } \\
\text { INAC.être_loin } \\
\text { dòy, } \\
\text { en-arrière } \\
\text { fàrá } \\
\text { endroit } \\
\text { sغ̀nć } \\
\text { dedans }\end{array}$ \\
\hline & \multicolumn{3}{|c|}{$\begin{array}{l}\text { Il y a un animal }{ }^{12} \text {, tu le perces et il part en courant, quand il est bien loin, alors } i \\
\text { revient et tu le tues à l'endroit même où tu l'avais percé. }\end{array}$} \\
\hline
\end{tabular}

\begin{tabular}{|c|c|c|c|}
\hline 8. & $\begin{array}{l}\text { Pám } \\
1 S \\
\text { ká } \\
\text { à_côté } \\
\text { Pạ́-ndèć } \\
\text { voilà_que. } \\
\text { 2P.D } \\
\text { zú } \\
\text { au- } \\
\text { dessus.D } \\
\text { Pá-ndè } \\
\text { voilà_que. } \\
\text { 2P.D } \\
\text { té } \\
\text { INAC.venir } \\
\text { Pà } \\
\text { 3S } \\
\text { bòyàyà } \\
\text { Npr }\end{array}$ & $\begin{array}{l}\text { tòkà } \\
\text { ACc.transpercer.D } \\
\text { zăy } \\
\text { continuité.D } \\
\text { ndàkà } \\
\text { ACC.pourchasser.D } \\
\text { dòtà } \\
\text { Npr } \\
\text { pèyá } \\
\text { ACC.revenir.D } \\
\text { gbé } \\
\text { INF.Acc.tuer } \\
\text { ká } \\
\text { à_côté } \\
\text { sí-dòy } \\
\text { à_nouveau }\end{array}$ & $\begin{array}{l}\text { bíá } \\
\text { aulacode } \\
\text { Gòyàyà, } \\
\text { Npr } \\
\text { sínć } \\
\text { en_s'en_allant } \\
\text { mè, } \\
\text { là-bas } \\
\text { dòy } \\
\text { en-arrière } \\
\text { ngbák } \\
\text { véritable } \\
\text { zăy } \\
\text { continuité.D }\end{array}$ \\
\hline & \multicolumn{3}{|c|}{$\begin{array}{l}\text { J'ai percé un aulacode sur les bords de 'Boyanga, nous l'avons poursuivi jusqu'à Dota } \\
\text { là-bas, et nous sommes revenus sur nos pas et sommes venus le tuer, lui, juste sur les } \\
\text { bords de 'Boyanga à nouveau. }\end{array}$} \\
\hline
\end{tabular}

Les devinettes gbaya définissent ces deux éléments en insistant sur le duo qu'ils forment tout en attribuant à chacun une nature propre bien différente, qui oppose une lourdeur immobile (marteau de forge, babouin) à une fluidité en mouvement (l'eau, la pluie). De plus, lorsque chacun est considéré dans sa spécificité, c'est en termes de gibier que ces chasseurs en parlent. Ainsi le pipi est un petit gibier qui entraîne le chasseur dans une course poursuite qui suit une boucle fermée le ramenant toujours à 
son point de départ. Le caca est, lui, un éléphant, le plus gros des animaux qu'ils connaissent, que le chasseur abandonne sur place, ne prenant même pas la peine de récupérer sa sagaie. La dégradation qui s'en suivra favorise bien sûr l'arrivée des mouches, qu'on ne manque pas de trouver à chaque fois sur tout animal qui se décompose à l'air libre. Ces devinettes ne cherchent pas à manifester un comique de situation, elles ne véhiculent pas de jugement de valeur, elles expriment la conceptualisation culturelle de ces deux éléments pour les Gbaya.

\section{Que nous apprennent les proverbes?}

Le proverbe tó-wèn littéralement une "parole pilée » dont il faut reconstruire le sens est employé pour porter un avis sur quelqu'un à propos d'une situation que seuls partagent ces deux personnes, ce qui évite le sentiment de honte que ne manquerait pas de produire une critique directe en langage clair qui pointe nécessairement celui qui est ainsi mis en cause (Roulon et Doko, 2009). Il s'agit d'un langage indirect qui contrairement aux devinettes n'est pas construit sur une image à interpréter mais bien sur une structure binaire dont les deux éléments ne semblent pas aller ensemble. Trouver ce qui les réunit fait apparaître ce qu'on veut exprimer (critique, désaveu, menace, etc.). Ceci, tous apprennent à le décrypter. Par contre le contexte d'application reste un savoir partagé seulement par les deux protagonistes : celui qui dit le proverbe et celui auquel il s'adresse, sans bien sûr le désigner explicitement. C'est un message qui, une fois reçu, force à réfléchir et donc demande toujours du temps : il n'y a jamais de réponse immédiate.

Dans les proverbes où seul est attesté le caca, il s'ajoute, aux points de vue physique et physiologique déjà exprimés de façon neutre par les devinettes, un point de vue plus dépréciatif qui cible négativement sa mauvaise odeur et la souillure qu'il produit.

\section{Point de vue neutre}

31 Si caca et pipi sont associés au moment de la défécation, c'est un autre automatisme qui est mentionné dans le proverbe suivant : l'érection involontaire qui est la seule que peut se permettre l'étranger-voyageur. Il s'agit d'un conseil pour dire à quelqu'un de faire profil bas :

\begin{tabular}{|l|l|l|l|}
\hline 9. & bǒr & gغ̀nغ̀ & kúr \\
& pénis.D & étranger \\
qùr & INAC.se_dresser \\
& caca & \\
\hline & au-dessus.D & \multicolumn{2}{|c|}{} \\
\hline \multicolumn{2}{|l|}{ Le pénis du voyageur se dresse au moment de la défécation. } \\
\hline
\end{tabular}

L'association déjà présentée entre le caca et la mouche est reprise par le proverbe suivant qui illustre une situation perçue comme une frustration ressentie par l'intéressé qu'on peut rendre par l'expression adverbiale 'tant pis pour moi' signalant qu'il renonce à ce qu'il convoitait : 


\begin{tabular}{|l|l|l|l|}
\hline \multirow{2}{*}{10.} & dǒr & dòó & yì \\
& caca.D & sous.D & eau \\
& bàá & tè & zí \\
& Acc.prendre.D & entité.D & mouche \\
\hline & Une merde sous l'eau ennuie la mouche. \\
\hline
\end{tabular}

La relation nécessaire et suffisante entre l'absorption de nourriture et l'excrément produit, exprimée par le proverbe suivant, symbolise un résultat donné comme un profit qui est unique à chaque fois. C'est une façon d'opposer un refus, en invitant le solliciteur à se contenter de ce qu'il a déjà eu :

\begin{tabular}{|l|l|l|l|}
\hline 11. & jóy & kòm乏̀ & só \\
& INJ.manger & de_sorte_que. & INAC.déverser \\
& dòráà & $2 S$ & zù \\
& caca.nom & Pá & sur \\
& bárá & INF.Acc.mettre & \\
& dalle_des_termites & & \\
\hline & Mange et tu en déféqueras la merde sur une pierre plate. \\
\hline
\end{tabular}

\section{Point de vue dépréciatif}

Nul ne sait de quoi l'avenir est fait. Ce que le français exprime par les expressions 'fontaine je ne boirai pas de ton eau' ou 'il ne faut jurer de rien' est exprimé en gbaya par le proverbe suivant où l'endroit souillé par la défécation devient le seul abri possible contre la pluie. Le personnage mis en scène est un étranger au pays gbaya, donc quelqu'un qui a priori n'avait pas à se soucier de ses traces, et pourtant !

\begin{tabular}{|c|c|c|c|}
\hline 12. & $\begin{array}{l}\text { mbùsà } \\
\text { haoussa } \\
\text { ká } \\
\text { alors } \\
\text { Pé } \\
\text { INF.ACC.poser } \\
\text { Péí } \\
\text { LOGS } \\
\text { Gò } \\
\text { encore } \\
\text { kórò } \\
\text { pluie } \\
\text { mbùsà } \\
\text { haoussa } \\
\text { káí } \\
\text { INAC.ramasser } \\
\text { Pá } \\
\text { INF.ACC.jeter } \\
\text { Pó } \\
\text { INAC.dormir }\end{array}$ & $\begin{array}{l}\text { kùrà } \\
\text { ACC.se_lever.D } \\
\text { só } \\
\text { INAC.déverser } \\
\text { kútùà, } \\
\text { maison } \\
\text { té-?ò } \\
\text { VIRT.INAC.dormir } \\
\text { ná. } \\
\text { NEG } \\
\text { ndák } \\
\text { INAC.chasser } \\
\text { péí } \\
\text { INAC.revenir } \\
\text { dòr } \\
\text { caca } \\
\text { táá } \\
\text { vers } \\
\text { kútùàí } \\
\text { maison.ANAPH }\end{array}$ & $\begin{array}{l}\text { sớ, } \\
\text { RÉV } \\
\text { dòr } \\
\text { caca } \\
\text { béè } \\
\text { INAC.pouvoir_être.INS } \\
\text { kútùàí } \\
\text { maison.ANAPH } \\
\text { ká } \\
\text { alors } \\
\text { mbùsà, } \\
\text { haoussa } \\
\text { dòy } \\
\text { en_arrière } \\
\text { kóí } \\
\text { de.LOGS } \\
\text { mغ̀ } \\
\text { là-bas } \\
\text { sí-dòy. } \\
\text { à_nouveau }\end{array}$ \\
\hline & \multicolumn{3}{|c|}{$\begin{array}{l}\text { Un Haoussa s'était levé et avait fait caca dans une maison se disant qu'il n'y dormirait plus. } \\
\text { Quand la pluie a chassé le Haoussa, le Haoussa est revenu, il a ramassé son caca en le jetant là- } \\
\text { bas et il a dormi à nouveau dans cette maison. }\end{array}$} \\
\hline
\end{tabular}


proverbe suivant invite à savoir dépasser une première impression de dégoût, rappelant que c'est l'aire de déjection de certains termites qui permet de repérer l'endroit où on pourra les récolter, ce qu'on fait bien sûr puisqu'ils sont comestibles et appréciés :

\begin{tabular}{|l|l|l|l|}
\hline 13. & tó & zó & Pá \\
& IMP.écraser & herbe & INF.Acc.mettre \\
& zú & dòr, & Pèì \\
& au-dessus.D & caca & et_donc.on \\
& jóy & dòè & \\
& INAC.manger & termite & \\
\hline & Écrase des herbes sur la merde (qui a permis de repérer la termitière) et mange-en \\
& les termites.
\end{tabular}

La mésaventure du chien racontée par le proverbe suivant est une mise en garde adressée à quelqu'un qu'on veut remettre à sa place, lui rappelant qu'il n'est pas étranger aux mauvaises actions dont il parle, car il oublie de voir sur lui-même ce qu'il dénonce chez les autres :

\begin{tabular}{|l|l|l|l|}
\hline 14 & tòyó & Pé & dòr \\
& chien & INAc.laisser & caca \\
& zù & ndál & ṅ̀ \\
& tête & INAc.lècher & INST \\
& kó & dàn & kéí \\
& celle & ami & de.LoGs \\
\hline \multicolumn{2}{|c|}{} \\
& Le chien laisse le caca sur sa tête et lèche néanmoins celui de son copain. \\
\hline
\end{tabular}

Contrairement aux devinettes, les proverbes ne parlent que du caca. Ce sont le plus souvent des expressions brèves qui peuvent parfois devenir un véritable petit récit comme en 12. Tous ces proverbes mettent en scène des situations de la vie quotidienne faciles à comprendre. On voit cependant à côté d'une présentation neutre de phénomènes physiques et physiologiques comparables à ceux qui étaient mis en scène dans les devinettes (ex. 9 à 11), émerger un point de vue dépréciatif qui cible la mauvaise odeur et la souillure, qui sont culturellement bien connues mais n'avaient pas été jusqu'ici mentionnées (ex.12 à 14). Par le biais des trois derniers proverbes, le locuteur instrumentalise les excréments pour motiver une réaction de répulsion en jouant sur leur aspect déplaisant : l'excrément y symbolise la chose dégoûtante.

\section{Que nous apprennent les contes?}

Contrairement aux proverbes qui ne parlent que du caca, les « contes» to comme les devinettes font intervenir caca et pipi. Cependant, tandis qu'ils sont assez équitablement pris en compte dans les devinettes, l'urine est bien moins souvent mise en scène que les excréments dans les contes. 


\section{Le pipi dans les contes}

39

neut bien sûr signaler la mention du pipi lorsqu'un personnage submergé par la peur, fait sous lui ou qu'un autre s'éloigne en prétextant 'aller en brousse' ce qui est, rappelons-le, l'expression consacrée pour dire « aller aux toilettes » :

\begin{tabular}{|c|c|c|c|}
\hline 15. & 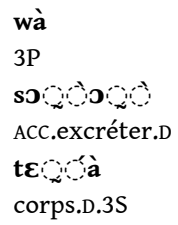 & $\begin{array}{l}\text { gùsáà, } \\
\text { ACc.frapper.D.3S } \\
\text { Pínì } \\
\text { pipi } \\
\text { wè̀̀ } \\
\text { irrépressiblement }\end{array}$ & $\begin{array}{l}\text { Pa__-nàá } \\
\text { voilà_que.3S } \\
\text { Pá } \\
\text { INF-ACC.poser }\end{array}$ \\
\hline & \multicolumn{3}{|c|}{ On l'a frappé, et il a uriné sur lui de façon irrépressible. } \\
\hline
\end{tabular}

Au-delà de ces manifestations tout à fait ordinaires, le pipi est aussi utilisé comme un motif. Il s'agit du procédé utilisé dans le conte du "Fromager des divinités", par l'enfant de la tortue qui, envoyé par sa mère chercher $\mathrm{du}$ feu chez Wanto ${ }^{13}$, éteint plusieurs fois de suite la torche de roseaux qu'il vient d'enflammer en urinant dessus afin de pouvoir y retourner et faire ainsi durer son séjour dans la maison de Wanto, le temps de découvrir ce qu'ils vont manger :

\begin{tabular}{|l|l|l|l|}
\hline 16. & bêm & kó & táná \\
& enfant & de & tortue \\
& bá & wèè & mó-gbò \\
& INAC.prendre & feu & INF.vIRTUEL.sortir \\
& zân, & sò̀̃ & Pínì \\
& dehors & Acc.excréter.D & bìyá, \\
& yíkáà, & wèè & Acc.s'éteindre \\
& surface.D.DEF & feu & \\
& sìi & dòy & en_arrière \\
& Acc.retourner.D & L'enfant de la tortue prend le feu pour sortir dehors, pisse dessus, le feu s'éteint et il y \\
\hline & retourne. \\
\hline
\end{tabular}

Dans une autre version de ce conte, c'est le père tortue lui-même qui va chercher du feu chez Wanto, il l'éteint alors en soufflant dessus.

Dans le conte "Des deux filles et la balançoire" (Roulon-Doko, 1992), le repas bâclé que préparent les deux filles pressées de pouvoir essayer la balançoire précise qu'elles ont choisi des feuilles de Justicia insularis au goût sucré peu apprécié, qu'elles les ont mal épluchées, n'y ont pas mis de sel et les ont accompagnées d'une boule de manioc toute molle dont la couleur rouge vif est caractérisée par référence à la couleur de l'urine :

\begin{tabular}{|c|c|c|c|}
\hline 17. & $\begin{array}{l}\text { hé } \\
\text { comme } \\
\text { só } \\
\text { REV } \\
\text { sò? } \\
\text { excréter.ÉNONCIATIF }\end{array}$ & $\begin{array}{l}\text { wà } \\
3 P \\
\text { Pínì } \\
\text { pipi } \\
\text { gá } \\
\text { comme }\end{array}$ & $\begin{array}{l}\text { sò̀̃ } \\
\text { Acc.excréter.D } \\
\text { sغ̀nć } \\
\text { dedans }\end{array}$ \\
\hline & \multicolumn{3}{|c|}{ Comme si elles avaient auparavant fait pipi dedans. } \\
\hline
\end{tabular}



mare des animaux". Après de nombreuses péripéties, Wanto ayant fait fuir Gbason ${ }^{14}$, repère ses réserves de maïs dans la partie supérieure d'un abri-grenier dont il est aussitôt précisé :

\begin{tabular}{|c|c|c|c|}
\hline 18. & $\begin{array}{l}\text { ká } \\
\text { alors } \\
\text { hớgó } \\
\text { comme_ça } \\
\text { sò } \\
\text { à_excréter } \\
\text { gbàsõ, } \\
\text { Npr } \\
\text { dór } \\
\text { caca }\end{array}$ & $\begin{array}{l}\text { đòò } \\
\text { sous.D } \\
\text { né } \\
\text { ÊTRE-ESS } \\
\text { dòr } \\
\text { caca } \\
\text { né } \\
\text { ETRE-ESS } \\
\text { hógó } \\
\text { comme_ça }\end{array}$ & $\begin{array}{l}\text { fâl } \\
\text { abri-grenier } \\
\text { fàrá } \\
\text { endroit } \\
\text { kó } \\
\text { de } \\
\text { zóm } \\
\text { énorme.D } \\
\text { dèkpè_dèkpè_dèkpè_dèkpè. } \\
\text { tout_mou.RD }\end{array}$ \\
\hline & \multicolumn{3}{|c|}{$\begin{array}{l}\text { Alors sous le grenier-abri comme ça c'est l'endroit à faire caca de Gbason (les WC de Gbason), } \\
\text { c'est une énorme quantité de merde comme une mer de boue. }\end{array}$} \\
\hline
\end{tabular}

47 Tout à l'idée de prendre des épis de maïs pour les manger en famille il monte sur la plateforme, et marchant imprudemment sur la couche de spathes étalées au sol, il passe au travers : 


\begin{tabular}{|c|c|c|c|}
\hline 19. & 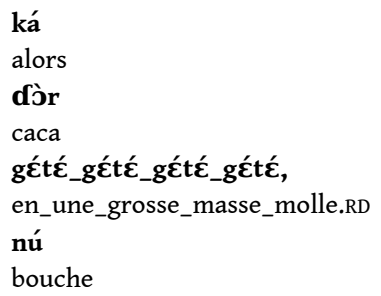 & $\begin{array}{l}\text { pèà } \\
\text { ACC.revenir.D } \\
\text { kó } \\
\text { de } \\
\text { yé } \\
\text { INAC.entrer } \\
\text { Pé. } \\
\text { déjà }\end{array}$ & $\begin{array}{l}\text { ték } \\
\text { INF.ACC.tomber } \\
\text { gbàsò } \\
\text { Npr } \\
\text { bór } \\
\text { INF.ACC.atteindre }\end{array}$ \\
\hline
\end{tabular}

Dans cette situation pour le moins inconfortable, il lance un appel à sa femme Naanombol qui ne manque pas d'accourir lui demandant ce qui se passe:

\begin{tabular}{|c|c|c|c|}
\hline 20. & $\begin{array}{l}\text { nàá-nòmbôl! } \\
\text { Npr } \\
\text { Pàrí } \\
\text { mettre_de_côté. } \\
\text { ENONCIATIF } \\
\text { dòrí } \\
\text { caca.ANAPH } \\
\text { Pám } \\
1 S \\
\text { Pám } \\
1 S \\
\text { kó } \\
\text { de } \\
\text { mò } \\
\text { chose } \\
\text { sínć. } \\
\text { en_partant } \\
\text { tĚm. } \\
\text { corps.D.1S } \\
\text { té-fè. } \\
\text { V.INAC.mourir }\end{array}$ & $\begin{array}{l}\text { Pár } \\
\text { IMP.mettre_de_côté } \\
\text { P乏́, } \\
\text { donc } \\
\text { yúné } \\
\text { en_ôtant } \\
\text { tèká } \\
\text { Acc.tomber.D } \\
\text { tèká } \\
\text { Acc.tomber.D } \\
\text { ó́y } \\
\text { méchant } \\
\text { năm } \\
\text { REL.1S.D } \\
\text { Pár } \\
\text { IMP.mettre_de_côté } \\
\text { en_ôtant } \\
\text { mí } \\
\text { 1S }\end{array}$ & $\begin{array}{l}\text { đò̀í } \\
\text { caca.ANAPH } \\
\text { Pár } \\
\text { IMP.mettre_de_côté } \\
\text { těm, } \\
\text { corps.d.1S } \\
\text { dòl15 } \\
\text { caca }^{15} \\
\text { dòl } \\
\text { caca } \\
\text { mà } \\
\text { IND. } \\
\text { ndàkà } \\
\text { Acc.poursuivre.D } \\
\text { yúné } \\
\text { en_ôtant } \\
\text { Pàm } \\
\text { 1S.suB }\end{array}$ \\
\hline & \multicolumn{3}{|c|}{$\begin{array}{l}\text { Naa-nombol! Ôte donc cette merde, ôte cette merde de mon corps! Je suis tombé dans de la merde. Je suis } \\
\text { tombé dans la merde de cette méchante chose que je poursuivais. Ôte-la de mon corps! Moi je vais } \\
\text { mourir. }\end{array}$} \\
\hline
\end{tabular}

\begin{tabular}{|c|c|c|c|}
\hline 21. & $\begin{array}{l}\text { Páá-nc̀ } \\
\text { voilà_que } \\
\text { té } \\
\text { à } \\
\text { gbàsò } \\
\text { Npr }\end{array}$ & $\begin{array}{l}\text { nàá-nòmbôl } \\
\text { Npr } \\
\text { dò̀r } \\
\text { caca } \\
\text { hغ̇.... } \\
\text { ce }\end{array}$ & $\begin{array}{l}\text { dùkà } \\
\text { ACc.rester.D } \\
\text { kó } \\
\text { de }\end{array}$ \\
\hline
\end{tabular}

Elle parvient à le dégager et à le tirer au sec. Wanto court aussitôt se laver dans la mare des oiseaux qu'il aperçoit non loin de là. Il se lave longtemps et, une fois sorti de l'eau, constatant que la goutte d'eau restée au coin de sa bouche est toute sucrée, il s'écrie : 


\begin{tabular}{|c|c|c|c|}
\hline 22. & $\begin{array}{l}\text { mò } \\
\text { chose } \\
\text { kó } \\
\text { de } \\
\text { tèká } \\
\text { Acc.tomber.D } \\
\text { jòká, } \\
\text { Acc.être_doux } \\
\text { ká } \\
\text { alors } \\
\text { mà } \\
\text { IND } \\
\text { gò } \\
\text { quoi }\end{array}$ & 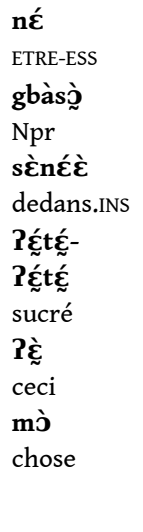 & $\begin{array}{l}\text { dòr } \\
\text { caca } \\
\text { năm } \\
\text { REL.1S. D } \\
\text { Pá-nc̀ } \\
\text { voilà_que } \\
\text { náà ! } \\
\text { DISC } \\
\text { né } \\
\text { ETRE-ESS } \\
\text { gà } \\
\text { comme.INTERROGATIF }\end{array}$ \\
\hline & \multicolumn{3}{|c|}{$\begin{array}{l}\text { Serait-ce la merde de Gbason dans laquelle je suis tombé qui est douce et vraiment très } \\
\text { sucrée, se demande-t-il ? Alors ça, c'est quelque chose comme quoi? }\end{array}$} \\
\hline
\end{tabular}

Et, s'étant léché le corps, il va puiser un peu d'eau et s'aperçoit qu'elle est sucrée comme du miel !...

Dans ce conte, l'engloutissement dans cette masse de merde fait rire et la tâche qui incombe à son épouse est particulièrement dégoûtante. Ceci rend encore plus cocasse le retournement de la situation lorsque se baignant dans l'eau sucrée de la mare des oiseaux, il se demande si ce pourrait être le goût des excréments de Gbason. La situation scabreuse dans lequel Wanto s'est retrouvé n'étonne personne, car il est coutumier du fait. Ne sachant résister au plaisir que promettent soit une bonne nourriture soit une danse, il accumule les situations qui pourraient être dramatiques s'il ne parvenait toujours à les retourner en sa faveur grâce à sa ruse, signe de son intelligence qu'il met d'ailleurs au service de tous. C'est ainsi qu'il est le héros civilisateur des contes gbaya. Représentant la complexité de chaque être humain, il a toujours l'indulgence et le soutien du public. Le conte qui suit ("Wanto qui vivait dans un fromager") est un bon exemple du comportement de ce personnage.

\section{Du caca à l'anti-nourriture}

Le terme d'anti-nourriture utilisé pour désigner un déchet qu'on propose comme une nourriture chez Aristophane (Jay-Robert, 2009, 149) est couramment employé dans des études sur les littératures orales (Görod-Karady, 1997,130) et spécifié comme "Les excréments et leur odeur nauséabonde symbolisent à la fois l'animalité, l'antinourriture, le poison, le mépris et la haine » (Blanchon, 1997, 194).

C'est le point de vue manifesté par le conte de "Wanto qui vivait dans un fromager". Gbason ayant découvert le mot de passe parvient à monter dans le fromager d'où il menace toute la famille de Wanto. Ce dernier parvient en rusant à l'en expulser mais se fait peu après capturer par Gbason qui tient alors à se venger. Pour ce faire, il le ligote dans une sacoche qu'il place sur une branche en haut d'un arbre en attendant de revenir avec du bois pour préparer le feu où il le cuira avant de le manger. Wanto appelle en vain tous ceux qui passent auprès de cet arbre pour se rendre à une fête dans le village voisin, mais tous refusent lorsqu'ils entendent le nom de Gbason. Enfin ymánpí-nù-gée dont le nom signifie littéralement « Casse et jette à terre sans raison » décide, lui, de détacher Wanto et de partir ensuite avec lui à la fête. Or il est alors précisé que : 


\begin{tabular}{|c|c|c|c|}
\hline 23. & $\begin{array}{l}\text { wàntò } \\
\text { Wanto } \\
\text { dò̀r } \\
\text { caca } \\
\text { gásá } \\
\text { grand } \\
\text { ymán-pí-nù-géè } \\
\text { Npr } \\
\text { sî-dòy, } \\
\text { à_nouveau } \\
\text { hěn } \\
\text { dans.D } \\
\text { gèrà } \\
\text { fromager } \\
\text { dòòrí } \\
\text { caca.ANAPH } \\
\text { gáí } \\
\text { INF-ACC.suspendre } \\
\text { gèrà } \\
\text { fromager }\end{array}$ & 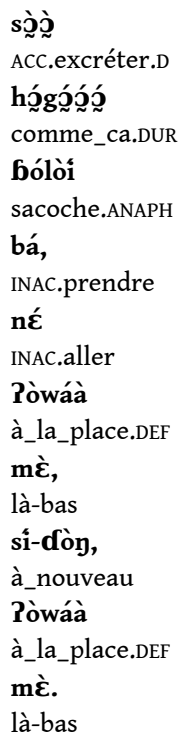 & $\begin{array}{l}\text { só } \\
\text { REV } \\
\text { dún } \\
\text { INF-ACC.remplir } \\
\text { hínńć. } \\
\text { saturé } \\
\text { yák } \\
\text { INAC.partir } \\
\text { gáí } \\
\text { INF-ACC.suspendre } \\
\text { ká } \\
\text { au_faîte.D } \\
\text { bá } \\
\text { INAC.prendre } \\
\text { né } \\
\text { INAC.aller } \\
\text { ká } \\
\text { au_faîte.D }\end{array}$ \\
\hline & \multicolumn{3}{|c|}{$\begin{array}{l}\text { Wanto avait tellement déféqué que la sacoche était pleine à ras-bord }{ }^{16} \text {. ymán-pí-nù-gée la } \\
\text { prend et retourne la suspendre à la place qu'elle occupait dans le fromager. Il reprend les } \\
\text { excréments et retourne les suspendre à la même place en haut de l'arbre. }\end{array}$} \\
\hline
\end{tabular}

Peu après Gbason revient et prépare un grand feu pour avoir beaucoup de braises. Il monte ensuite à l'arbre et décroche la sacoche qu'il redescend et jette dans le feu, ça grésille. Vous savez bien que ce sont des excréments qui remplissent la sacoche, précise le conteur à l'intention de l'auditoire. C'est lourd, aussi lourd que Wanto. C'est vraiment lourd. La sacoche reste donc dans le feu à brûler, entourée de flammes. Gbason se réjouit du sort de Wanto :

\begin{tabular}{|c|c|c|c|}
\hline 24. & 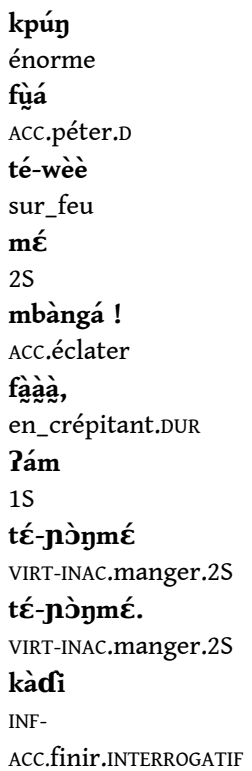 & $\begin{array}{l}\text { ká } \\
\text { alors } \\
\text { kò } \\
\text { intérieur.D } \\
\text { mè. } \\
\text { là-bas } \\
\text { zòká } \\
\text { ACC.voir.D } \\
\text { nóí } \\
\text { INAc.bouillir } \\
\text { mbàngà } \\
\text { ACc.éclater.D } \\
\text { tòáà } \\
\text { ACc.dire.D.INS } \\
\text { sósóò. } \\
\text { aujourd'hui } \\
\text { yílkmé } \\
\text { œil.2S } \\
\text { Pè } \\
\text { déjà }\end{array}$ & $\begin{array}{l}\text { đór } \\
\text { caca } \\
\text { bólò } \\
\text { sacoche } \\
\text { mé ! } \\
2 S \\
\text { zànmé } \\
\text { ventre.2S } \\
\text { nèné } \\
\text { INST } \\
\text { sí-dòn. } \\
\text { à_nouveau } \\
\text { Pám } \\
\text { 1S } \\
\text { Pám } \\
\text { 1S } \\
\text { mbàngà } \\
\text { Acc.éclater.D }\end{array}$ \\
\hline & \multicolumn{3}{|c|}{$\begin{array}{l}\text { Bruyamment les excréments pètent dans la sacoche sur le feu là. Toi, t'as vu ton ventre a } \\
\text { éclaté! Ça continue à bouillir en crépitant, ça éclate à nouveau. Je l'ai bien dit que je te } \\
\text { mangerai aujourd'hui. Je vais te manger. Tes yeux n'ont-ils pas déjà éclaté? }\end{array}$} \\
\hline
\end{tabular}



provoquer sa belle-famille et précipiter de façon spectaculaire le dénouement. En effet, non contente de refuser d'accompagner la femme du frère aîné de son mari dans ses activités, elle se révèle gourmande mais surtout souille toute la maison en faisant caca partout :

\begin{tabular}{|c|c|c|c|}
\hline 26. & $\begin{array}{l}\text { Pèé } \\
\text { Puis.D } \\
\text { dòrrr } \\
\text { caca.DuR } \\
\text { kpánààà, } \\
\text { poterie.DUR } \\
\text { Pòé. } \\
\text { aussi } \\
\text { dòr } \\
\text { caca } \\
\text { Pòé. } \\
\text { aussi }\end{array}$ & $\begin{array}{l}\text { Pà̀ } \\
\text { 3S } \\
\text { hár } \\
\text { INF.ACC.aligner } \\
\text { ngbàrà } \\
\text { entre.D } \\
\text { Pà } \\
\text { 3S } \\
\text { hár } \\
\text { INF.ACC.aligner }\end{array}$ & $\begin{array}{l}\text { sớ } \\
\text { INAC.excréter } \\
\text { ngbàrà } \\
\text { entre.D } \\
\text { kútùà } \\
\text { maison } \\
\text { s0- } \\
\text { INAC.excréter } \\
\text { sènć } \\
\text { dedans }\end{array}$ \\
\hline & \multicolumn{3}{|c|}{$\begin{array}{l}\text { Puis elle fait des cacas qu'elle aligne au milieu des poteries, au milieu de la maison } \\
\text { aussi. Elle fait des cacas qu'elle aligne dedans aussi. }\end{array}$} \\
\hline
\end{tabular}



premier signe d'alerte est la présence de mouches évoquées ici par leur bourdonnement :

\begin{tabular}{|c|c|c|c|}
\hline 27. & 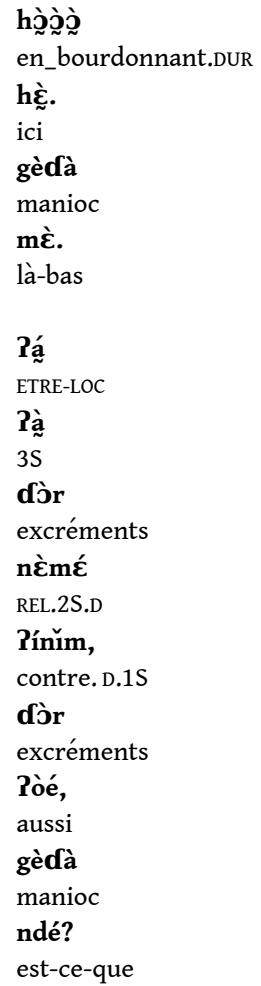 & 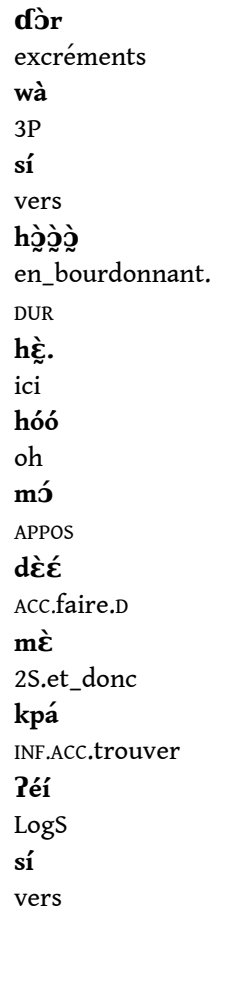 & $\begin{array}{l}\text { Pá } \\
\text { ETRE-LOC } \\
\text { bá } \\
\text { INAC.prendre } \\
\text { kútùà } \\
\text { maison } \\
\text { dòr } \\
\text { excréments } \\
\text { sá } \\
\text { mais } \\
\text { zóyá-zòrò } \\
\text { Npr } \\
\text { P̀̀ } \\
\text { celui-ci } \\
\text { bòò } \\
\text { donc } \\
\text { só } \\
\text { INAC.excréter } \\
\text { kútùà } \\
\text { maison } \\
\text { té-Pè } \\
\text { VIRT.INAC.poser } \\
\text { dí-gè } \\
\text { où }\end{array}$ \\
\hline & \multicolumn{3}{|c|}{$\begin{array}{l}\text { Des mouches bourdonnent, il y a du caca ici. Elles portent le manioc dans la maison là-bas. Des } \\
\text { mouches bourdonnent, il y a du caca ici. Mais oh! Fille-poisson ce caca que voici que tu as bien } \\
\text { sûr fait pour m'ennuyer, tu as fait caca partout dans la maison aussi, où vais-je poser le } \\
\text { manioc? }\end{array}$} \\
\hline
\end{tabular}

60 L'épouse, en s'entendant appeler "fille-poisson », déclare aussitôt qu'elle retourne auprès des siens. Au retour du mari qui ne voyant plus sa femme demande des explications, la fillette de son frère aîné commence ainsi :

\begin{tabular}{|c|c|c|c|}
\hline \multirow[t]{2}{*}{28.} & $\begin{array}{l}\text { Pówà, } \\
\text { dis_donc } \\
\text { pèà } \\
\text { Acc.revenir.D } \\
\text { mè } \\
\text { ce_là-bas } \\
\text { nàá-yè } \\
\text { maman } \\
\text { Pá } \\
\text { INF-ACc.poser } \\
\text { bźt_fśt_bét } \\
\text { entièrement.DUR }\end{array}$ & $\begin{array}{l}\text { bààbáà, } \\
\text { père } \\
\text { tè } \\
\text { à } \\
\text { wó } \\
\text { vraiment } \\
\text { sò̀ } \\
\text { AcC.D.xcréter.D } \\
\text { ngbàrà } \\
\text { entre } \\
\text { Pòé. } \\
\text { aussi }\end{array}$ & $\begin{array}{l}\text { Péć } \\
1 \mathrm{P} \\
\text { gèdà } \\
\text { manioc } \\
\text { ká } \\
\text { alors } \\
\text { dòr } \\
\text { excréments } \\
\text { kpánà } \\
\text { poterie }\end{array}$ \\
\hline & $\begin{array}{l}\text { Écoutez, papa, nou } \\
\text { partout partout ent }\end{array}$ & $\begin{array}{l}\text { entrées du ma } \\
\text { ssi. }\end{array}$ & it fait caca \\
\hline
\end{tabular}


61 Dans ce cas, l'attitude provocante de la nouvelle épouse diminue la responsabilité de l'épouse du frère aîné, ce qui n'est pas le cas dans les autres versions de ce même conte $^{17}$ où la volonté de transgresser l'interdit semble n'avoir aucune autre justification que le refus de ce mariage particulier. Contrairement aux deux contes précédents, le personnage de la fille-poisson utilise volontairement ses excréments pour produire une souillure.

\section{Le caca thème d'un conte}

Dans le conte de "Pourquoi l'eau tue les gens", la défécation est plus qu'un épisode, elle est au centre même du conte. Il s'agit d'un aigle qui a fait son nid au sommet d'un fromager au milieu d'une rivière. Devant aller chercher de la nourriture pour ses deux petites, la mère aigle ${ }^{18}$ leur fait la recommandation suivante :

\begin{tabular}{|c|c|c|c|}
\hline 29. & 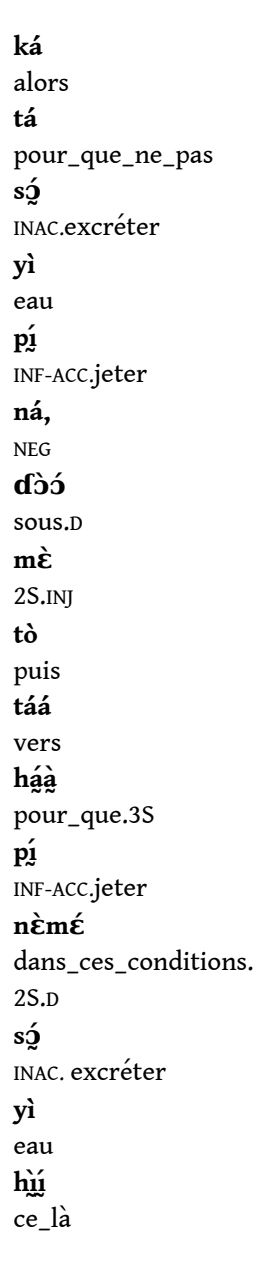 & 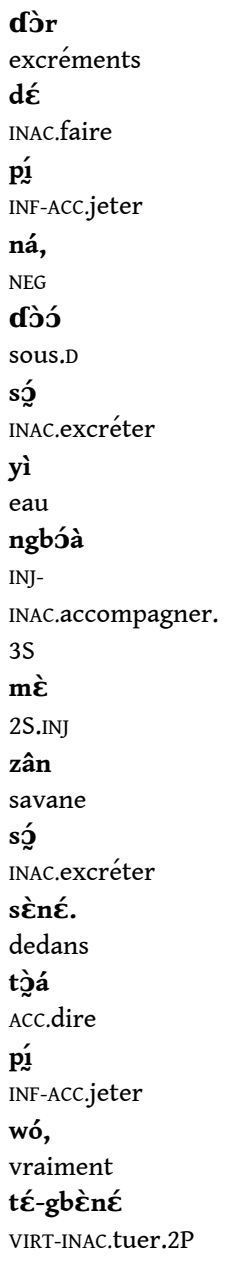 & 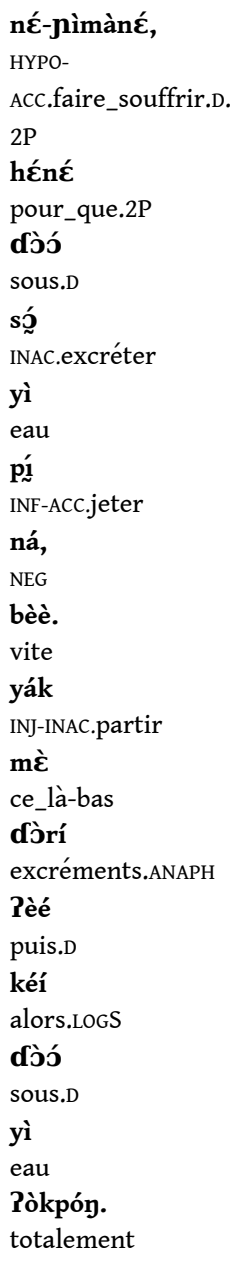 \\
\hline & $\begin{array}{l}\text { Quand vous avez envie } \\
\text { faire caca dans l'eau. Ac } \\
\text { si tu dis que tu fais vrair }\end{array}$ & $\begin{array}{l}\text { faut pas faire cacad } \\
\text { Puis, va dans la brou } \\
\text { u, cette eau-là vous }\end{array}$ & $\begin{array}{l}\text { re caca dans l'eau, pas } \\
\text { u'elle y fasse caca. Car } \\
\text { ux. }\end{array}$ \\
\hline
\end{tabular}

63 Ayant bien expliqué qu'elle part très loin chercher de la nourriture, elle s'en va. Peu après : 


\begin{tabular}{|l|l|l|l|}
\hline 30. & tíkídí & bé & jáíí \\
& un_peu & petit & frère/ \\
& Pó & dàà! & sœur.ANAPH \\
& POL & soeur_aînée & dòr \\
& Pà̀ & jìmáà & excréments \\
& 3 mí & $1 \mathrm{~S}$ \\
\hline & Acc.faire_souffrir.INS & La toute petite sœur dit : «Sceur aînée! J'ai très envie de faire caca. » \\
\hline
\end{tabular}

La sœur aînée réagit aussitôt et propose de l'emmener sur la terre ferme, mais c'est trop tard :

\begin{tabular}{|l|l|l|l|}
\hline 31. & Péì & Pó & dàà \\
& zut & POL & soeur_aînée \\
& Pám & sò̃ & pí \\
& $1 S$ & INAc.excréter & INF-Acc.jeter \\
& dòó & yì & Pè \\
& sous.D & eau & déjà \\
\hline \multicolumn{2}{|l|}{} \\
\hline
\end{tabular}

La mère revient une première fois à temps, mais la seconde fois est fatale aux enfants qui sont engloutis par l'eau. Et de conclure que c'est depuis ce jour-là que l'eau peut tuer des gens :

\begin{tabular}{|c|c|c|c|}
\hline 32. & $\begin{array}{l}\text { Pá } \\
\text { voilà_que } \\
\text { kpók } \\
\text { le_même } \\
\text { s’̀̀̀ } \\
\text { Acc.excréter.D } \\
\text { yì } \\
\text { eau } \\
\text { yì } \\
\text { eau }\end{array}$ & 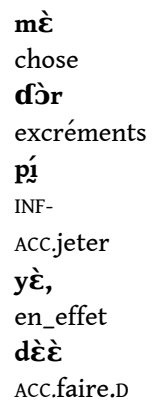 & $\begin{array}{l}\text { né } \\
\text { ETRE-ESS } \\
\text { nàá } \\
\text { REL.3S.D } \\
\text { dòó } \\
\text { sous.D } \\
\text { sá } \\
\text { mais } \\
\text { gbéyéć } \\
\text { INF-ACC.tuer. } \\
\text { 1P }\end{array}$ \\
\hline & Et c'est en effe & a fait dar & eau nous tue. \\
\hline
\end{tabular}

De fait personne ne fait jamais caca dans l'eau des petites rivières qui sillonnent le terroir gbaya, ni même au bord de l'eau ${ }^{19}$ bien qu'en dehors de ce conte cela ne soit jamais explicitement prescrit comme tel.

67 Il convient de souligner que la construction signifiant 'avoir envie d'aller à la selle' utilise un verbe de sensation, le verbe transitif « faire souffrir » nim qui a comme sujet le stimulus $d \grave{r}$ et comme complément l'expérient. Cette construction qui souligne l'absence de contrôle de ce dernier sur ce phénomène serait mieux rendue par le terme 'besoin' qu'on emploie d'ailleurs quand en français on parle d'aller faire ses besoins'. Dans le cas de l'urine en particulier, cette absence de contrôle joue certainement un rôle dans le fait que le pipi au lit n'est jamais réprimandé, même si on a des remèdes pour le soigner. Ainsi quand un enfant déjà grand est atteint d'énurésie, il n'est pas l'objet de moqueries, mais on attend de lui qu'il prenne en charge l'exposition au soleil quotidienne de la natte sur laquelle il dort. Par contre la construction utilisée pour dire 
'faire caca ou pipi', n'utilise pas un verbe de sensation, mais un verbe ordinaire transitif

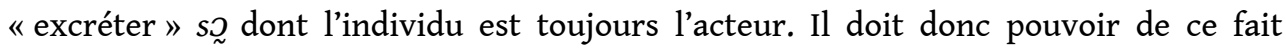
contrôler à volonté le processus. C'est d'ailleurs en ces termes que l'exigence d'un contrôle est posée par l'aigle, ou que la fille-poisson manifeste sa volonté de provoquer sa belle-famille, ou encore que l'enfant de la tortue urine intentionnellement sur la torche pour l'éteindre.

Dans ces trois cas, le personnage est celui qui agit, il a l'initiative du procès. Ainsi, contrairement aux devinettes ou aux proverbes, dans les contes c'est cette mise en scène volontaire du pipi comme du caca qui est exploitée.

\section{Conclusion}

$\mathrm{Au}$ terme de ce parcours qui a permis d'appréhender la conceptualisation gbaya du domaine du scatologique, il ressort que le vocabulaire utilisé a le plus souvent une valeur notionnelle beaucoup plus large (déchet, excréter, AA qualificatifs, etc.) sans connotations qui le réduirait au seul domaine scatologique comme cela se produit en français dont Michel Renaud ${ }^{20}$, parlant de Rabelais, précise :

... le scatologique, discours sur le déchet, l'immondice, discours immonde, est comme son objet - difficilement récupérable. Il marque une régression, procède de préoccupations dégradantes, d'obsessions infantiles ou séniles et, dans le même temps, il rétablit une coïncidence intolérable entre le signe et la chose signifiée. Il conjugue la trivialité du vocabulaire, la complaisance verbale et la référence excrémentielle.

Le vocabulaire gbaya est neutre et tout au long de cette présentation on a pu voir les difficultés rencontrées pour traduire le plus fidèlement possible ces termes en français où chaque contexte d'emploi pousse à choisir le registre qui semble le mieux adapté. Ce faisant, cette 'surcharge' sémantique n'est pas conforme à l'expression du gbaya qui n'a pas de vocabulaire scatologique spécifique.

L'utilisation de ce vocabulaire dans les devinettes exprime la conception que les Gbaya en ont, pointant les spécificités de chacun d'entre eux, sans faire intervenir un jugement de valeur. Celles-ci donnent seulement à voir des images qu'il convient d'identifier. Dans les proverbes, au-delà des images déjà présentées, un jugement dépréciatif apparaît qui cible la mauvaise odeur des excréments et la souillure qui va avec. Le caca devient alors l'élément type qui va provoquer le dégoût sur lequel le proverbe invite à réfléchir voire à le dépasser. Dans les contes, le pipi mais surtout le caca deviennent des éléments que les personnages mettent en scène pour créer des situations comiques, exploitant en particulier pour ce dernier ses côtés dépréciatifs.

\section{BIBLIOGRAPHIE}

BERTAUD du CHAZAUD, Henri, 1971, Nouveau dictionnaire des synonymes, Paris, Hachette-Tchou. 
BLANCHON, Flora, 1997, Enfances, Paris, Presses Universitaires de Paris-Sorbonne, coll. « Asie ».

FLAUBERT, Gustave, 1964, Les œuvres de Gustave Flaubert, t. VII : Correspondance 1853-1856,

Lausanne, Éditions Rencontre.

GÖRÖG-KARADY, Veronika, 1997, Univers familial dans les contes africains : liens du sang, liens d'alliance, Paris, L'Harmattan.

JAY-ROBERT, Ghislaine, 2009, L'invention comique : Enquête sur la poétique d'Aristophane, Besançon, Presses Universitaires de Franche Comté.

Renaud, Michel, De l'invective à la jubilation. Le scatologique dans l'œuvre de Rabelais. Résumé d'un article publié dans Cahiers Textuel, n 34/44, S.T.D., Université Paris 7, $n^{\circ}$ 4/5, 1989.

REY, Alain (dir.), 1994, Dictionnaire historique, Paris, Le Robert, Édition du Club France-Loisirs.

ROULON-Dоко, Paulette, 1992, Traduction d'un conte gbaya : Les deux filles et la balançoire,

L'immature, 4, septembre-novembre, p. 46-47.

ROULON-Dоко, Paulette, 2004, Les devinettes en pays gbaya, in P. Boyeldieu et P. Nougayrol (éds),

Terrains d'Afrique, Hommage à F. Cloarec-Heiss, Louvain, Peteers, p. 121-135.

ROULON, Paulette et Dоко, Raymond, 2009, La parole pilée : accès au symbolisme chez les Gbaya

'bodoé de Centrafrique, Cahiers de Littérature Orale, $\mathrm{n}^{\circ}$ 66, Paris, Publications Langues'O, p. 217-232.

[ $1^{\text {ère }}$ éd. 1983, Cahiers de Littérature Orale, $n^{\circ} 13$, p. 33-49].

\section{NOTES}

1. Dans cet article, il y a différents niveaux de guillemets : "chevrons » pour les traductions françaises des termes étrangers, 'simples' pour les différentes entrées, expressions, qualificatifs et autres et "doubles" pour les citations.

2. Les Gbaya sont une population d'environ un demi-million de personnes qui occupent un territoire situé pour les quatre cinquième à l'ouest de la République Centrafricaine et pour le dernier cinquième au centre est du Cameroun. Le groupe numériquement le plus important appelé 'Gbaya kara' ou 'Gbaya du Nord' est subdivisé en plusieurs sous-groupes dont font partie les Gbaya 'bodoé chez lesquels je travaille. Ceux-ci forment un groupe homogène d'environ cinq mille personnes réparties en une quarantaine de villages au sud-ouest de Bouar (R.C.A.).

3. Bien distinct du terme pàyà " détritus, ordure » qui désigne les déchets végétaux.

4. Qui réfère au bas-ventre.

5. Le méconium dont on souligne la couleur très noire est appelé en gbaya tárí ou đつ̌r-dìnj̀ $(\sim /$ arbre sp.) car il peut contenir des petites boulettes qui rappellent les fruits de cet arbre.

6. Les abréviations utilisées dans le mot à mot sont: ACC accompli ; ANAPH anaphorique ; APPOS appositif; D déterminant tonal; DEF défini ; DUR duratif ; ESS essentiel ; INAC inaccompli ; INF infinitif; IMP impératif ; INS intensif; INST instrumental ; LOC locatif; LOG logophorique ; NEG négation ; Npr nom propre ; PL pluriel ; REL relatif ; REV révolu ; RD intensif ; SUB subordinatif ; VIRT virtuel ; et pour pronoms personnels 1, 2, 3 selon les personnes suivi de S pour le singulier et de $\mathrm{P}$ pour le pluriel.

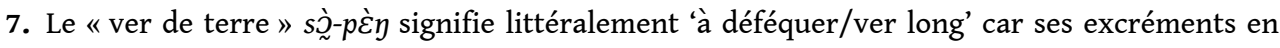
forme de tortillons ressemblent à de longs vers.

8. Fiente globuleuse plus ou moins dure de certains animaux (TLF).

9. Matière fécale (de l'homme ou de certains animaux) consistante et moulée (TLF). 
10. Ce terme peut désigner aussi bien le beau-père que la belle-mère, que les deux, ce qu'en français local les gens rendent par le terme « beau ou beaux » selon les cas.

11. Il existe d'ailleurs un verbe «faire gicler, projeter » $d \mathcal{\varepsilon} r$ qui s'applique spécifiquement à l'urine.

12. C'est la formulation la plus courante pour introduire les devinettes.

13. Wanto est le héros civilisateur des contes. Son nom qui signifie littéralement le « maître des contes » désigne également l'araignée. C'est un personnage complexe, homme, divinité et araignée.

14. Gbason, littéralement la "grande divinité » est un personnage qui accapare tous les biens que Wanto systématiquement récupère pour en faire profiter tous les hommes.

15. Parler particulier de Wanto qui remplace les ' $r$ ' par des 'l' comme dans le parler enfantin qu'on emploie pour parler aux petits enfants afin qu'ils acquièrent bien la position de la langue du son 'l' avant de pouvoir produire un 'r' à un seul battement.

16. C'est bien sûr sous l'effet de la peur, mais cela n'est pas explicitement indiqué, comme cela l'était pour le pipi comme signalé plus haut.

17. Il s'agit alors de la mère du garçon, sa belle-mère qui nomme sa belle-fille "fille poisson » sans aucune provocation de sa part.

18. Il s'agit d'une mère et de ses deux fillettes aigle, ce qui n'est linguistiquement perceptible qu'une fois le conte déjà bien avancé, avec l'emploi de "sœur aînée » parallèlement au terme «frère/sœur » ne pouvant référer alors qu'à un ego féminin.

19. Alors que certaines populations pensent l'eau comme l'endroit adéquat pour faire leurs besoins.

20. De l'invective à la jubilation. Le scatologique dans l'œuvre de Rabelais, PDF 130219 de l'École Alsacienne.

\section{RÉSUMÉS}

Ayant présenté tout d'abord les termes gbaya à la disposition des locuteurs et leurs emplois courants dans la vie quotidienne, j'étudie la conception gbaya de ce domaine par l'étude des représentations qu'ils en ont, recourant pour cela à l'examen des devinettes, des proverbes puis des contes. Il ressort que ce sujet est traité en gbaya avec un vocabulaire unique, le plus souvent non spécifique, qui échappe à la hiérarchisation qui s'impose en français entre scientifique, médical, familier ou vulgaire voire grossier.

In this article the author presents Gbaya terms at the disposal of speakers and their use in daily life. She then studies Gbaya perceptions of this domain by analyzing how it is represented through, for example, riddles, proverbs, and tales. The outcome of her study shows that the Gbaya treat this subject with a unique vocabulary, most often non specific, which escapes from the hierarchical distinction governing the French language between the scientific, the medical, the familiar, the vulgar, and the coarse. 
INDEX

Keywords : Pee, Poop, Scatology, Gbaya, Linguistic Registers, Riddles, Central African Republic, Tale

Index géographique : République centrafricaine

nomsmotscles Gbaya

Thèmes : anthropologie (Afrique)

Mots-clés : pipi, conte, scatologie, niveaux de langue, proverbe 